\title{
Image Restoration Based on Quadtree Data Structures
}

\author{
Yanhua Ma \\ Qingdao University of Science and Technology, China \\ yhma@qust.edu.cn
}

\begin{abstract}
This paper investigates the problem of image restoration when the image is corrupted by Pepper-and-salt noise and proposes a new restoration algorithm based on quadtree data structures. The crucial parts of the algorithm are picking noise and fuzzy estimation, so that unlike some other usual denoising methods, it avoids blurring and averaging edges and non-noise pixels. This paper describes, analyzes and compares several methods and results of removing noise from image. Experimental results indicate that the new algorithm proposed by this paper is superior.
\end{abstract}

Keywords: Image restoration, image reconstruction, image denoising, fuzzy estimation, Quadtree Data Structures

\section{Introduction}

Image restoration is not only an old but also still a problem discussed. Aggelos K. Katsaggelos in [1] writes that the field of digital image restoration is concerned with the reconstruction or estimation of uncorrupted images from noisy, blurred ones. This blurring may be caused by optical distortions, object motion during imaging, or atmospheric turbulence. There are existing or potential applications of image restoration in many scientific and engineering fields, such as aerial imaging, remote sensing, electron microscopy and medical imaging. At the same times, [2] tell us that the purpose of image restoration is to "compensate for" or "undo" defects which degrade an image. Degradation comes in many forms such as motion blur, noise, and camera misfocus. Image restoration attempts to reconstruct or recover an image that has been degraded by using a priori knowledge of the degradation phenomenon . W. Jun etc. in[3] proposes denoising method based on Wavelet threshold.

Data and an algorithm are the two basic related parts of any program. Data organization often considerably affects the simplicity of the selection and the implementation of an algorithm, and the choice of data structures is therefore a fundamental question when writing a program [4]. Traditional image data structure such as matrices, graphs, lists of object properties, and relational databases are important not only for direct representation of image information, but also as a basis for more complex hierarchical method of image representation [5].

Image denoising is the important studying content in image restoration. There are a lot of the ways proposed, see [6-10]. It is generally recognized that image denoising should be the first step of any image processing method. An important task of mathematical image processing is image denoising, see [10]. The contents include the noise removing from various images, such as satellite and medical images, by various methods. An example is the recent image denoising research for brain MR in [11]. In typical images the noise can be modeled with either a Gaussian ("normal"), uniform, or pepper-and-salt ("impulse") distribution, see [12]. 


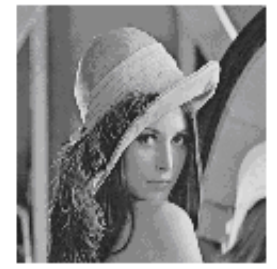

(a1)

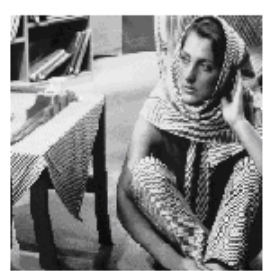

(b1)

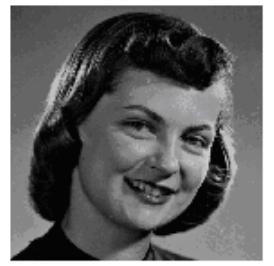

(c1)

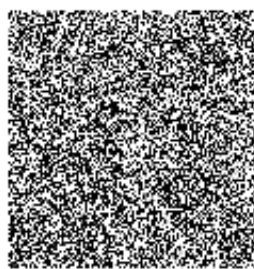

(a2)

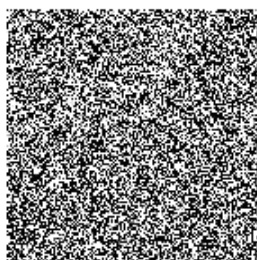

(b2)

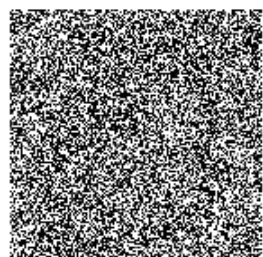

(c2)

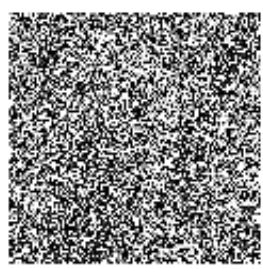

(a3)

(a6)

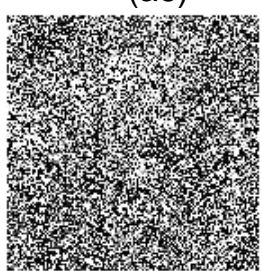

(b3)

(b6)

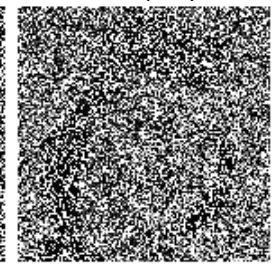

(c3)

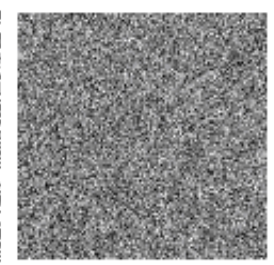

(a4)

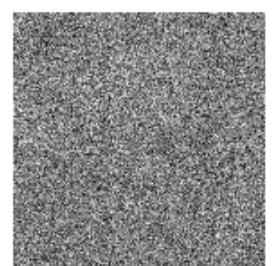

(b4)

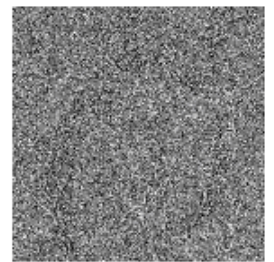

(c4)

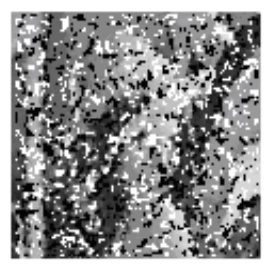

(a5)

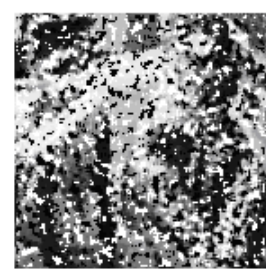

(b5 )

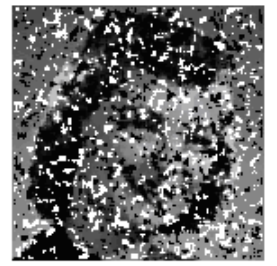

(c5)

(c6)

Figure 1. Images with higher density noise and filters

(a1), (b1) and (c1) original images without noise;

(a2),(b2) and (c2) images with $90 \%$ noise density corresponding to the left column;

(a3--c3) images restructured by median filter with size $3 \times 3$;

(a4--c4) images restructured by mean filter with size $3 \times 3$;

(a5--c5) images restructured by median filter with size $11 \times 11$;

(a6--c6) images restructured by mean filter with size $11 \times 11$.

The predominant method to suppress the noise in an image is filtering. The ordinary filtering is mean filtering and median filtering. However, whether using mean filtering or using median filtering the quality of the denoised image is obviously decline with the noisy density increasing, see (a3-c3) and (a4-c4) in Figure 1. Although enlarging the size of filtering region is able of enhancing the effect of denoising noise, it brings out the serious problem of blurring sharp edges in the image, see (a5-c5) and (a6-c6) in Figure 1. Indeed, the traditional denoising methods are already difficulty to reconstruct an image with higher noisy density, see Figure 1. In this paper, we proposed a new denoising algorithm, which is combining quadtree data structures with fuzzy estimation. Using it can reconstruct an image from higher pepper-and-salt noisy density. The crucial parts of it are picking noise and fuzzy estimation. Experimental results indicate that the new method proposed by this paper is superior.

\section{Theory and Algorithm}

In this section, we discuss the algorithm based on quadtree data structures and fuzzy estimation.

Because the image to be analyzed is completely submerged in noise, a new and effective algorithm must be adopted. To mining out useful information, considerable quantities of image data must be processed and the feature of image data and processing algorithm must be considered. One very natural spatial relation in an 
image data is the neighborhood relation. The usual processing method based on neighborhood operation is mean filtering. However, as we already mentioned above, the mean filtering and median filtering are of no effect when the noisy density is very high. Median filtering can only pick the individual noise and mean filtering average only the noise instead of eliminating noise. Hierarchical data structures make it possible to use algorithms which decide a strategy for processing on the basis of relatively small quantities of data[5]. quadtree structure is one of hierarchical data structures. An advantage of image representation by means of quadtrees is the existence of simple algorithms for addition of images, computing object areas, and statistical moments.

Nothing than the large amount of data to be processed is more important for image when the processing plan has been decided. Therefore, this paper proposes a restoration algorithm based on quadtree data structure. The algorithm is as following.

\section{A. Picking Noise}

Assume $N \times N$ represent the sizes of the image, gray-scale range from black to white $[0, L], I(x, y)$ represents the image to be processed and $O(x, y)$ denotes the image picked noise. According to the feature of pepper-and-salt noise, the processing procedure can be presented by the following equation:

$$
O=F(I)=\left\{\begin{array}{lc}
I & I \in(0, L) \\
\phi & \text { otherwise }
\end{array}\right.
$$

The gray relation between $O$ and $I$ is showed by the following Figure 2.

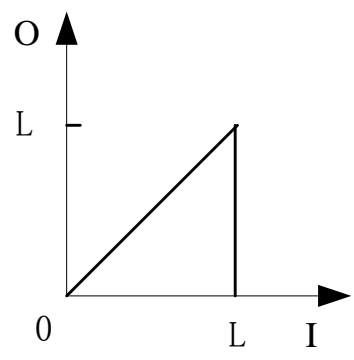

\section{Figure 2. The Gray Relation between $O$ and $I$}

\section{B. Quadtrees}

Quadtrees are modifications of T-pyramids. The define of T-pyramids is given in[5]. Every node of the quddtree except the leave is a parent node and has four children.

Figure 3 is an example of a simple quretree. 

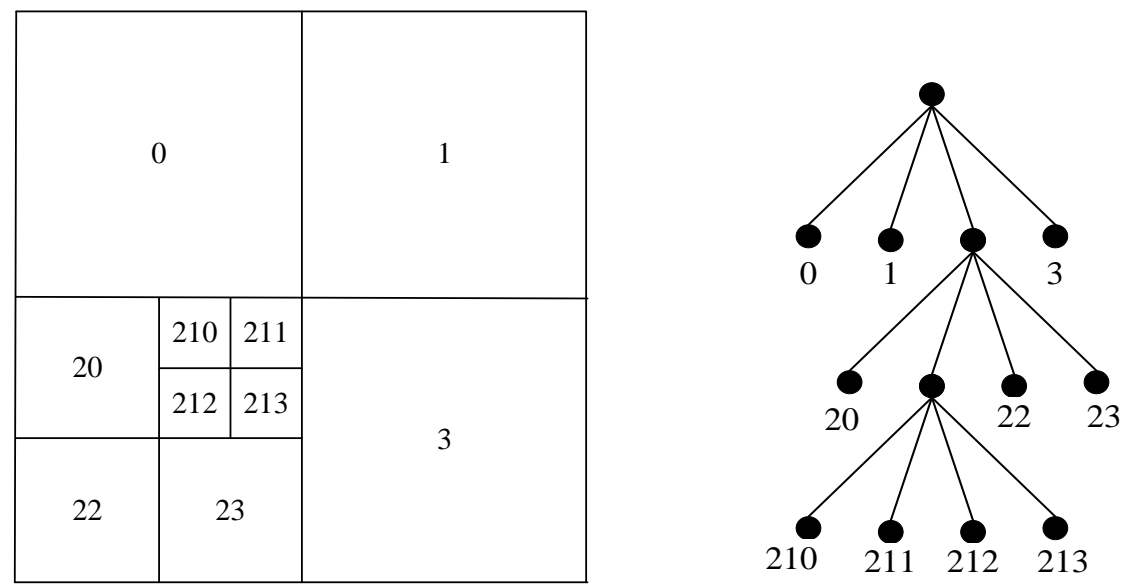

Figure 3. Quadtree

If a leave node(child) has value $\phi$,

make it possible to use algorithms which decide a strategy for processing on the basis of relatively small quantities of data. They work at the finest resolution only with those parts of the image for which it is essential, using knowledge instead of brute force to ease and speed up the processing. Quadtree is one of the typical structures of quadtree data structures.

\section{Fuzzy estimation algorithm}

To retrieval an image is to reestimate the noise pixels. According to the quadtree above, values of individual nodes of the tree are defined by the function $V$. Values of the leaf nodes are the same as values of the image function (brightness) in the original image at the finest resolution, such as nodes $210,211,212,213$, except they are the noise pixels. Values of nodes in other levels of the tree are a fuzzy estimation of $2^{M-k}$ child nodes.

The fuzzy estimation consists of several main steps that are outlined in the following algorithm.

1. Inputs and Outputs: the inputs of the fuzzy estimation system are the image picked noise, such as the image $O$ in section 1, and the outputs are to be estimated values of the noise pixels which are $\phi$ in the image $O$.

2. Defining the fuzzy set used for estimating pixel $(i, j)$ :

$$
S_{i j}(1)=\{P(m, n)\}
$$

where $m=[i, i+1]$ if $i$ is odd,

$$
m=[i-1, i] \text { otherwise } i \text {; }
$$

$n=[j, j+1]$, if $j$ is odd,

$n=[j-1, j]$, otherwise $j$;

$\& \operatorname{Value}(m, n) \notin \phi . S_{i j}(1)$ is an uncertain set of the four child nodes of the parent with child node $(i, j)$

3. Computing

$$
\operatorname{Value}(i, j)=\operatorname{Mean}\left(V\left(S_{i j}(1)\right)\right)
$$

if $S_{i j}(1) \notin \phi$, where Mean $\left(V\left(S_{i j}(1)\right)\right)$ is the mean of image function (brightness) of all the nodes(leaf nodes) in the fuzzy set $S_{i j}(1)$.

4. If $S_{i j}(1) \in \phi$, then compute

$$
S_{i j}(\alpha)=\{P(m, n)\},
$$


$\alpha$ must be the least number which make $S_{i j}(1) \notin \phi . r_{i}=\operatorname{Mod}\left(i / 2^{\alpha}\right), \quad$ if $r_{i}=0$ then $r_{i}=2^{\alpha}$, Mod is the function to compute the remainder, $\operatorname{Value}(m, n) \notin \phi$,

$m=\left[i-r_{i}+1, i-r_{i}+2^{\alpha}\right], \quad n=\left[j-r_{j}+1, j-r_{j}+2^{\alpha}\right]$,

the definition of $r_{j}$ is similar to that of $r_{i} . S_{i j}(\alpha)$ is the fuzzy set of $\alpha^{\text {th }}$ level child nodes(leaf nodes) of $\alpha^{\text {th }}$ level parent of node $(i, j)$. In fuzzy set $S_{i j}(\alpha)$, there are at the least one $(m, n)$ that makes Value $(m, n) \notin \phi$.

5. Computing

$$
\operatorname{Value}(i, j)=\operatorname{Mean}\left(V\left(S_{i j}(\alpha)\right)\right)
$$

Furthermore, for all the child nodes $(x, y)$ of $(\alpha-1)^{\text {th }}$ level parent of node $(i, j)$, have

$$
\operatorname{Value}(x, y)=\operatorname{Value}(i, j) \text {. }
$$

This will reduce the processing time greatly. The proof is as follows.

Theorem1. Consider the image with size $N \times N$,

$\forall(i, j), \quad i, j \in[1, N]$,

$\exists \alpha \in Z, Z$ is the integer set.

makes $S_{i j}(\beta) \in \phi$, if $\beta \in[1, \alpha-1]$;

$$
S_{i j}(\beta) \notin \phi, \quad \text { if } \beta \in[\alpha, \Gamma] \text {. }
$$

If $(x, y) \in \Omega, \Omega$ is the set of all leaf nodes with the same $(\alpha-1)^{\text {th }}$ parent node as $(i, j)$,

$\exists \lambda \in Z$,

makes $S_{x y}(\eta) \in \phi$, if $\eta \in[1, \lambda-1]$;

$$
S_{x y}(\eta) \notin \phi, \quad \text { if } \eta \in[\lambda, \Gamma] \text {. }
$$

Then $\lambda \equiv \alpha$, i.e. $S_{x y}(\lambda)=S_{i j}(\alpha)$.

Where $\Gamma$ makes $2^{\Gamma}=N$.

Proof: If $\lambda \neq \alpha$, then $\lambda>\alpha$ or $\lambda<\alpha$

assume $\lambda<\beta$, then

$\exists(u, v),(u, v) \in$ the set of all the child nodes

(leaf nodes) of $\lambda^{\text {th }}$ level paregatiof nodaku, $\left.(u), v\right) \notin \phi$

makes Value $(u, v) \notin \phi, \quad \therefore S_{i j}(\alpha-1) \notin \phi$, this conflicts the condition provided.

$\because(u, v)$ and $(x, y)$ possess the samehthereferth, $\equiv \alpha$, vice verse.

$\therefore(u, v)$ and $(i, j)$ possess the same $(\alpha-1)^{\text {th }}$ parent

\section{Multi-image Processing}

When several images with noise can be used for operating simultaneously, assume there are $q$ sheets of image, then $O_{i}=F\left(I_{i}\right), i=1,2, \cdots q$,

$$
O=O_{1} \cup O_{2} \cup \cdots \cup O_{q}
$$

This will be helpful to improve the quality of image restored.

\section{Experimental Results}

Through the whole experiment, All the images possess the size $N \times N$ and the gray-level 256. The images without noise and the images with noise correspond (a1), (a2), (b1), (b2), (c1) and (c2) in Figure 1.repectively. In the Figure 4, the images at 
the left column are the restructured images by one noise image, and at the right column those are the restructured by four sheets of noise image.

\section{Discussions}

Compared the results of experiment, by Figure 4 and Figure 1, it is obvious that the algorithm proposed by this paper is more effective than the usual mean and median filtering. It is able of to restructure an image with 90 percents noise. Especially, when several noisy images are used for operating simultaneously, the effect of restored image is improved greatly.

\section{Conclusions}

This paper proposes a new de-noising algorithm based on hierarchical data structures combining with fuzzy estimation. Because it picks the noise pixels in advance, noise will not affect the restoration values. In addition, the fuzzy estimation deals with the noise pixels only so that no-noise pixels is changed. Therefore, the algorithm overcomes the disadvantages of traditional de-noising methods which are that de-nosing brings on blurring edges. Experimental results indicate that the new algorithm is superior.

However the algorithm isn't efficient for other noise, such as Gaussian noise. We are planning to improve the algorithm or to investigate a novel algorithm that can be effective for the images with other types of noise.

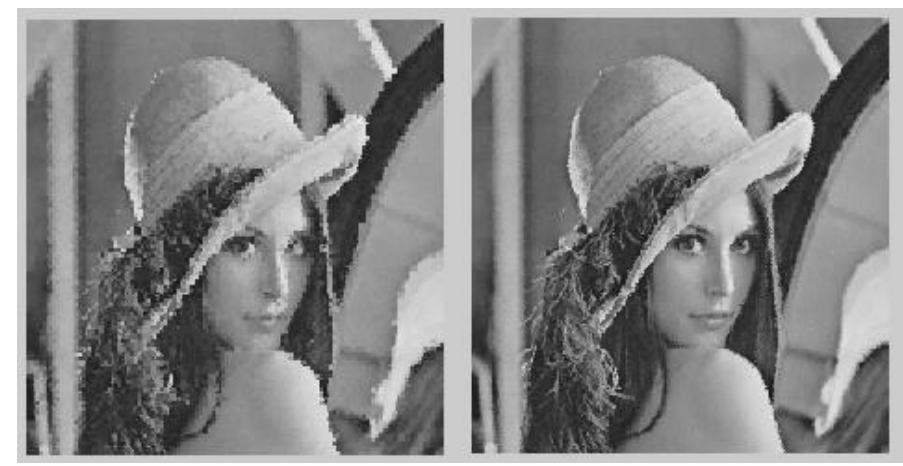

(a1)

(a2)

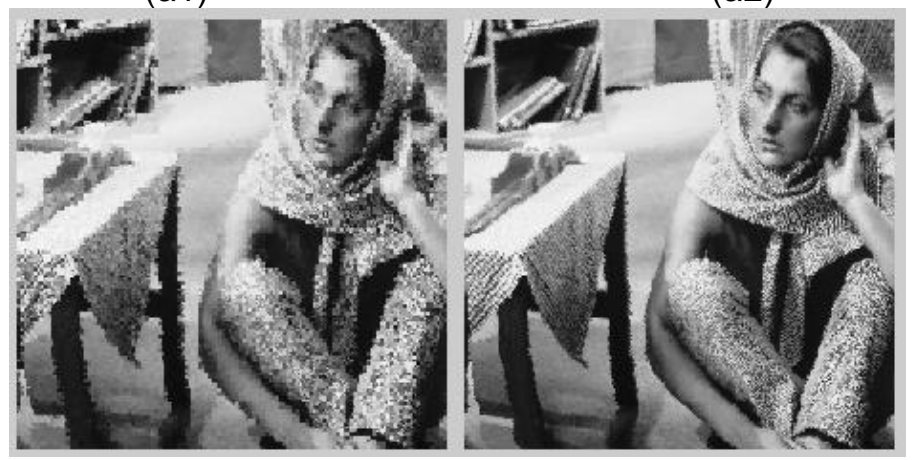

(b1)

(b2) 


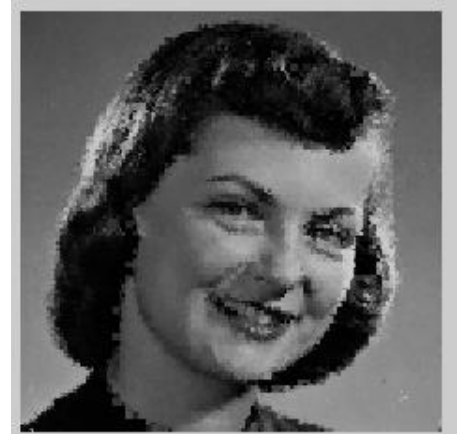

(c1)

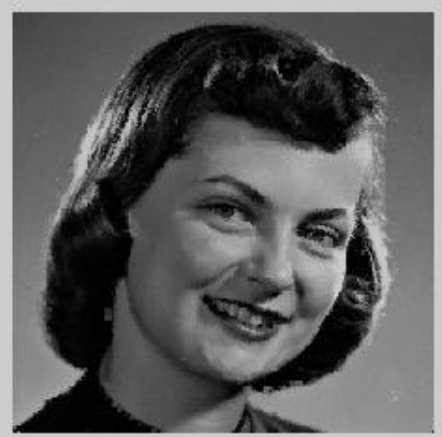

(c2)

\section{Figure 4. The De-noising Effect by the New Algorithm}

\section{References}

[1] A. K. Katsaggelos, "Digital Image Restoration (Springer Series in Information Sciences)", Springer-Verlag (1991).

[2] http://www.owlnet.rice.edu/ elec539/Projects99/BACH/proj2/intro.html.

[3] W. Jun, H. Z. Xiao and H. R. Jiong, "Research and Application of Denoising Method Based on Wavelet Threshold", 2010ICIECS, (2010) December 25-26, Wuhan, China.

[4] N. Wirth, "Algorithms + Data Structures = programs", prentice-Hall, Englewood Cliffs, NJ (1976).

[5] M. Sonka, V. Hlavac and R. Boyle, "Image processing, Analysis, and Machine Vision, Second Edition", Reprint for People's of China, Beijing (2002).

[6] J. S. Lee, "Digital image enhancement and noise filtering by use of local statistics", IEEE PAMI, vol. 2, no. 2, (1980), pp. 165-168.

[7] R. A. Peters II, "A new algorithm for image noise reduction using mathematical morphology", IEEE Transactions on Image Processing, vol. 4, no. 5, (1995), pp. 554-568.

[8] S. G. Chang, B. Yu and M. Vetterli, "Spatially adaptive wavelet thresholding with context modeling for image denoising", IEEE Transactions on Image Processing, vol. 9, no. 9, (2000), pp. 1522-1530.

[9] H. G. Senel, R. A. Peters II and B. M. Dawant, "Topological median filters Image Processing”, IEEE Transactions on Image Processing, vol. 11, no. 2, (2002), pp. 89-104.

[10] T. Le, R. Chartrand and T. J. Asakiz, "A variational approach to reconstructing images corrupted by Poisson noise", UCLA CAM Report, 05-49, (2005), September 21.

[11] A. Phophalia, "Rough set based image denoising for brain MR images", Signal Processing, vol. 103, (2014), pp. 24-35.

[12] L. Hargaš, M. Hrianka and A. Duga, "NOISE IMAGE RESTORATION BY SPATIAL FILTERS', http://www.urel.feec.vutbr.cz/ra2007/archive/ra2003/papers/376.pdf

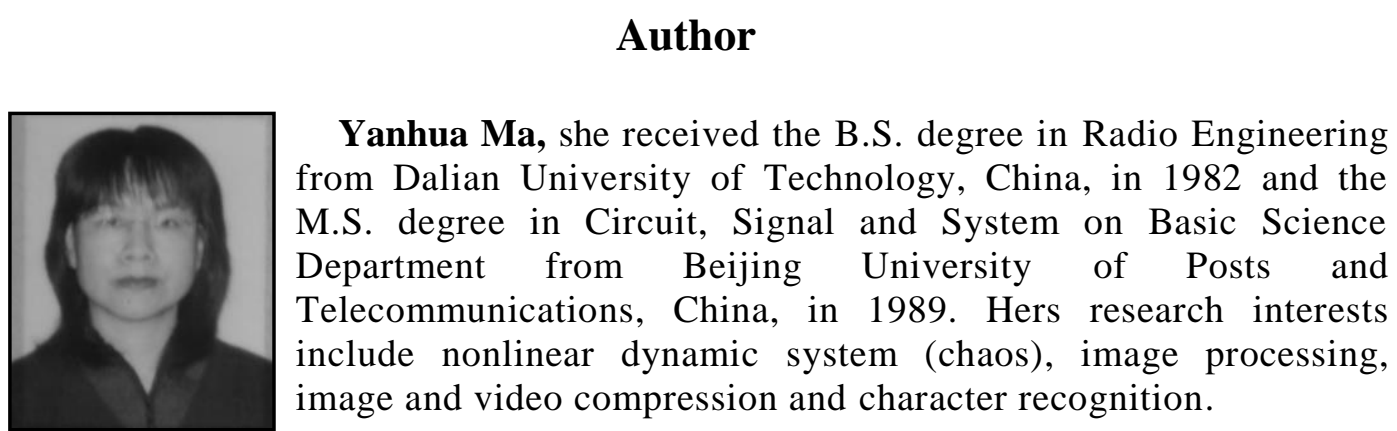


International Journal of Signal Processing, Image Processing and Pattern Recognition Vol.8, No.7 (2015) 